\title{
Multiplane-induced widening of stacking faults in fcc metals
}

Article in Applied Physics Letters · October 2007

DOI: $10.1063 / 1.2799246$

CITATIONS

3

5 authors, including:

Mutasem A. Shehadeh

American University of Beirut

30 PUBLICATIONS 264 CITATIONS

SEE PROFILE

\section{Zhengzheng Chen}

California State University, Northridge

44 PUBLICATIONS 370 CITATIONS

SEE PROFILE
READS

36

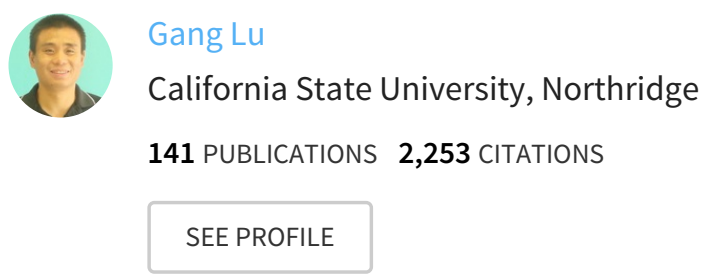

Nicholas Kioussis

California State University, Northridge

192 PUBLICATIONS 2,270 CITATIONS

SEE PROFILE

Some of the authors of this publication are also working on these related projects:

Project

Spin Transfer Torques View project

Project

Towards a Safer Design of Bio-inspired Helmets View project

All content following this page was uploaded by Nicholas Kioussis on 29 September 2017.

The user has requested enhancement of the downloaded file. 


\title{
Multiplane-induced widening of stacking faults in fcc metals
}

\author{
Mutasem A. Shehadeh, ${ }^{\text {a) }}$ G. Lu, Z. Chen, and N. Kioussis \\ Department of Physics and Astronomy, California State University, Northridge, \\ California 91330-8268, USA \\ N. M. Ghoniem \\ Department of Mechanical and Aerospace Engineering, University of California, Los Angeles, \\ California 90095-1597, USA
}

(Received 20 June 2007; accepted 25 September 2007; published online 24 October 2007)

\begin{abstract}
Using an ab initio-based parametric dislocation dynamics approach we show that Shockley partials on successive glide planes greatly assist the widening of stacking faults (SFs) in $\mathrm{Al}$ and $\mathrm{Ag}$. This effect is amplified when all trailing partials are pinned. Subsequent placement of Shockley partials on adjacent planes enhances further the widening of the SF width. In sharp contrast, dislocations with zero net Burgers vector across three successive planes form very compact cores in both $\mathrm{Al}$ and Ag, in agreement with recent experiments. (C) 2007 American Institute of Physics.
\end{abstract}

[DOI: $10.1063 / 1.2799246]$

Nanocrystalline (nc) metals exhibit mechanical properties, which are markedly superior to those of conventional materials, such as dramatically enhanced strength and hardness, ${ }^{1,2}$ which can coexist, in a few cases, with very good ductility. ${ }^{1-3}$ These exceptional properties have been attributed to their unique deformation mechanisms, which may be different from those in their coarse-grained (CG) counterparts. $^{4-7}$ These include partial dislocation emission from grain boundaries (GBs), deformation twinning (DT), full dislocation gliding, and GB sliding. The ratio $\gamma_{\mathrm{ut}}$ $-\gamma_{\text {isf }} / \gamma_{\text {us }}-\gamma_{\text {isf }}$ provides a measure of the competition between deformation twinning and full slip in fcc metals, where $\gamma_{\text {us }}$ is the unstable stacking fault energy required to nucleate the leading partial on the first glide plane, and $\gamma_{\text {isf }}$ and $\gamma_{\mathrm{ut}}$ are the intrinsic stacking fault (ISF) and unstable twin fault energies, ${ }^{8,9}$ respectively. The numerator (denominator) represents the energy barrier to nucleate the twining (trailing) partial on the adjacent (same) glide plane in the presence of the leading partial.

Molecular dynamics (MD) simulations, using extremely high strain rates in the order of $10^{6}-10^{8} \mathrm{~s}^{-1}$ predict that $\mathrm{nc}$ Al deforms via Shockley partial dislocation emission from GBs. ${ }^{10-12}$ Consequently, these partials form DT and partialdislocation-enclosed SF ribbons within the grains, the socalled homogeneous twinning mechanism. ${ }^{10}$ Interestingly, the observed SFs were found to be much wider (1.5-11 times) than both the experimental value in $\mathrm{CG} \mathrm{Al}$ and the theoretical value predicted by atomistic simulations. Such wide SFs are unexpected because Al has very high ISF energy. On the other hand, in nc metals $(\mathrm{Cu}$ or $\mathrm{Ag})$ with low $\gamma_{\text {isf }}$, no SF ribbons within the nanograins have been observed, while SFs across whole grain are ubiquitous. ${ }^{13}$ These wide SFs are attributed to both the small grain size and the presence of high stress concentration in nc metals. ${ }^{14,15} \mathrm{An}$ other twinning mechanism predicted by MD simulations ${ }^{10}$ and recently confirmed by experiment ${ }^{13}$ is the heterogeneous one in which the twins are nucleated on GBs.

\footnotetext{
${ }^{\text {a) }}$ Author to whom correspondence should be addressed. Electronic mail: mutasem@csun.edu
}

An intriguing question is the orientation of the Burgers vector of the trailing partial with respect to that of the leading partial that generated the SF. ${ }^{15}$ The trailing partials are generally believed to have identical Burgers vector, the socalled monotonic activation of partials. However, recent experiments ${ }^{16}$ find evidence of a twinning mechanism in nc metals, the so-called random activation of partials (RAPs) on successive (111) planes, which produce a zero net macrostrain, i.e., $\Sigma_{i=1}^{3} \mathbf{b}^{(i)}$. In this letter, we use an $a b$ initio-based parametric dislocation dynamics (DD) approach ${ }^{17}$ to investigate the effect of multiplanes on the SF width in $\mathrm{Al}$ and $\mathrm{Ag}$, with different ISF energies. We demonstrate that identical partials greatly assist the widening of SFs, especially in lowISF materials. This effect becomes further amplified when all trailing partials are pinned. We find that subsequent placement of leading partials on successive planes acts as a cascade process in enhancing further the widening of SFs of the previously placed partials. In sharp contrast, the dislocation configuration of zero macrostrain has very compact core in both $\mathrm{Al}$ and $\mathrm{Ag}$, consistent with recent experiments.

The approach is an extension ${ }^{17-19}$ of the Peierls-Nabarro model to multiplanes, where $n$ full dislocations nucleate on successive (111) slip planes, the dislocation spreading over all planes is explicitly accounted for, and both edge and screw components of the atomic displacements are treated on an equal footing. This allows for the dislocation dissociation into Shockley partials and utilizes the entire generalized stacking fault surface (GSFS) to capture the essential features of the core structure. ${ }^{17,19}$ Each full dislocation is represented by a set of $N$ dislocations with fractional Burgers vector having both edge and screw components. The net force on each fractional dislocation comprises of the elastic forces from all other fractional dislocations on all slip planes, the lattice restoring force across the glide plane derived from the GSFS, and the externally applied Escaig stress. The equilibrium structure of the dislocation core is obtained by seeking the equilibrium configuration of these fractional dislocations in all slip planes, using a simple viscous damping equation. The $a b$ initio calculation of the GSFS was carried out using the projector augmented-wave method, ${ }^{20}$ as implemented in the VASP code. ${ }^{21}$ The ISF energy for $\mathrm{Al}$ is $0.166 \mathrm{~J} \mathrm{~m}^{-2}$ compared to $0.030 \mathrm{~J} \mathrm{~m}^{-2}$ in $\mathrm{Ag}$. 


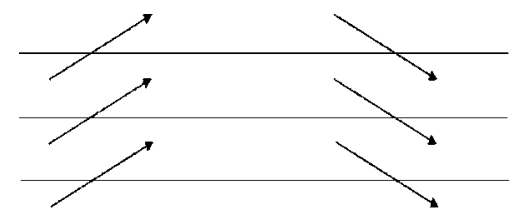

(a)

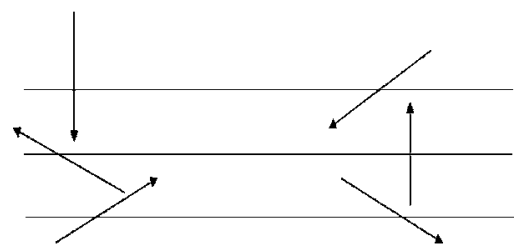

(b)

FIG. 1. Schematic configurations of edge dislocations on three successive (111) slip planes. (a) All dislocations have identical Burgers vectors. (b) Dislocations have different burgers vectors such that $\sum_{i=1}^{3} \mathbf{b}^{(i)}=0$.

In Fig. 1(a), we show the configuration of three full edge dislocations on the successive slip planes having identical Burgers vectors, i.e., all leading partials have $\mathbf{b}_{p}^{L}=a / 6[2 \overline{1} \overline{1}]$ and all trailing partials have $\mathbf{b}_{p}^{L}=a / 6[1 \overline{2} 1]$. In Fig. 1(b), we show the configuration corresponding to the RAP DT mechanism, where the three full dislocations have different Burgers vectors with the constraint that $\sum_{i=1}^{3} \mathbf{b}_{i, p}^{L}=\sum_{i=1}^{3} \mathbf{b}_{i, p}^{T}=0$.

Figure 2(a) shows the results for the partial separation on the first slip plane $d_{1}^{(n)}$ in $\mathrm{Al}$ as a function of the applied Escaig stress normalized to its critical value $\tau_{c}$ for a single (squares), two (circles), and three (triangles) identical $\left(b_{1}=b_{2}=b_{3}\right)$ edge dislocations placed on a single, two, and three successive slip planes respectively, $(n=1-3)$. The same Escaig stress is applied on each slip plane. The corresponding results for $\mathrm{Ag}$ are displayed in Fig. 2(b). Here, $\tau_{c}=2 \gamma_{\text {isf }} / b_{p}$ is the critical stress at which the partial separation becomes infinite for a single glide plane. ${ }^{22}$ This expression is derived using a simple model where the width of the ISF is determined by the balance between the elastic repulsion among the partials, the constant lattice restoring force (CLRF), and the external stress. $\tau_{c}$ is $2.0 \mathrm{GPa}(0.25 \mathrm{GPa})$ for $\mathrm{Al}(\mathrm{Ag})$. For comparison, we also display in Figs. 2(a) and

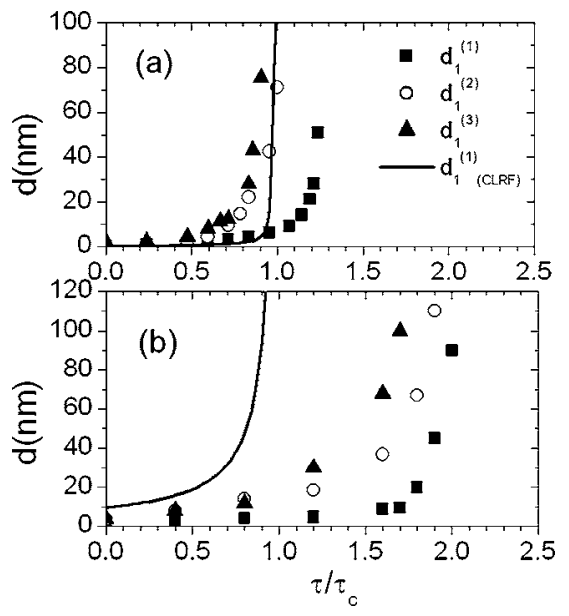

FIG. 2. Partial separation $d_{1}^{(n)}$ on the first slip plane for (a) $\mathrm{Al}$ and (b) Ag vs applied Escaig stress normalized to $\tau_{c}$ for a single (squares), two (circles), and three (triangles) identical edge dislocations placed on a single, two, and three successive slip planes. The symbols (curves) denote the results of the

hybrid DD-ab initio (CLRF) approach.
Downloaded 05 Dec 2007 to 130.166.113.235. Redistribution subject to AlP license or copyright; see http://apl.aip.org/apl/copyright.jsp

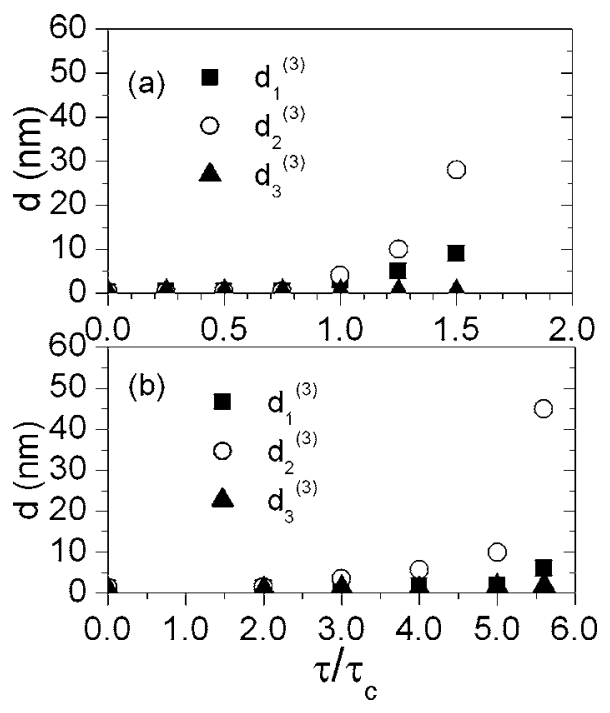

FIG. 3. Partial separation $d_{i}^{(3)}, i=1,2,3$ on the first, second, and third slip planes for (a) Al and (b) Ag vs applied Escaig stress normalized to $\tau_{c}$ for three dislocations placed on successive slip planes, where $b_{1}+b_{2}+b_{3}=0$.

2(b) the results for $d_{1}^{(1)}$ obtained from the simple CLRF model.

These results demonstrate that the formation of leading and trailing partials on the second and third slip planes greatly assists in increasing the ISF width on the first plane. This effect is due to the elastic repulsion among the fractional dislocations on the $n=2$ and $n=3$ planes with those on the first plane. Consequently, for a given grain size, the presence of twinning partials on multiple slip planes reduces considerably the applied stress needed to move the leading partials across the grain. The partial separation assistance from the third plane is smaller (larger) in $\mathrm{Al}(\mathrm{Ag})$. It is interesting to note that when the applied stress lies in the range of 0.7-1.2 GPa, suggested by experiment, the partial separation is between 5 and $10 \mathrm{~nm}$ in $\mathrm{Al}$ and larger than $200 \mathrm{~nm}$ in $\mathrm{Ag}$. These results are consistent with the experiment, ${ }^{2}$ where partial-dislocation-enclosed SF ribbons have been observed within the grains in nc Al. The SF width in Al will increase by about an order of magnitude $(\approx 100 \mathrm{~nm})$ under stress concentration of $\approx 2 \mathrm{GPa}$ found by MD simulations in the vicinity of SFs. ${ }^{23}$

In Fig. 3, we show the partial separation $d_{i}^{(3)}(i=1-3)$ on the first, second, and third planes for (a) Al and (b) Ag as a function of the Escaig stress normalized to $\tau_{c}$, for the case of three nonidentical dislocations shown in Fig. 1(b), with $\sum_{i=1}^{3} \mathbf{b}^{(i)}=0$. Interestingly, this configuration of Burgers vector, which corresponds to zero net macroscopic strain, results in a very compact core structure in both low- and high-ISF materials. In this case, the full dislocations on the first, second, and third planes are pure edge, $-30^{\circ}$, and $150^{\circ}$ character, respectively. Thus, the negative edge components of the second and third dislocations are elastically attracted with the positive edge of the first one, resulting in a compact core. The partial separation remains smaller than $5 \mathrm{~nm}$ for $\tau \leqslant \tau_{c}=2.0 \mathrm{GPa}$. Even for high stress of $\tau \approx 3.0 \mathrm{GPa}=1.5 \tau_{c}$, the largest partial separation is $\approx 20 \mathrm{~nm}$, much smaller than the corresponding value for the identical Burgers vector configurations in Fig. 2(a). We find similar trends in Ag, where, for example, an Escaig stress of $\tau=1.0 \mathrm{GPa}=4 \tau_{c}$ leads to a small partial separation of $40 \mathrm{~nm}$ on the second plane. These results are in agreement with recent experiments ${ }^{15}$ which in-
to AlP license or copyright; see http://apl.aip.org/apl/copyright.jsp 


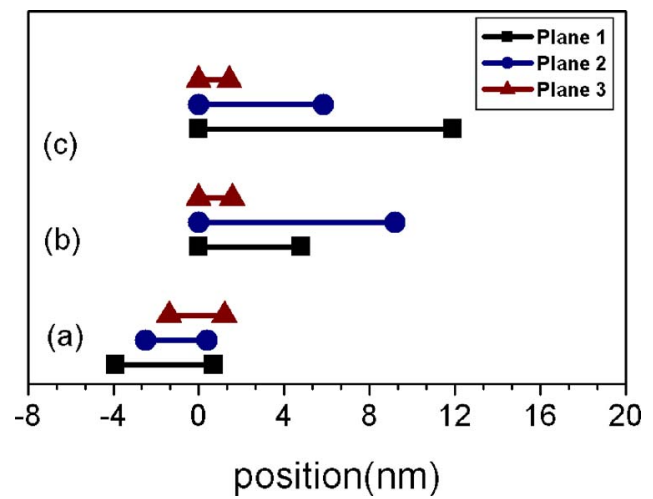

FIG. 4. (Color online) The equilibrium positions of the Shockley partials of three identical full dislocations nucleated on three successive slip planes in $\mathrm{Al}$, for an external stress of $\tau=1 \mathrm{GPa}$. (a) All partials nucleate simultaneously and are allowed to relax. (b) All partials are placed simultaneously and the trailing partials are pinned at the origin. (c) Leading partials are placed at different times.

dicate that DT in CG systems have partials of identical Burgers vectors, while the RAP configurations become the DT mechanism in nc materials.

In Fig. 4, we display the equilibrium configurations of the leading and trailing Shockley partials for three identical dislocations formed on the three adjacent slip planes in $\mathrm{Al}$ for an external stress of $\tau=1.0 \mathrm{GPa}$. The results correspond to the following: (a) homogeneous twinning in coarsegrained Al (Fig. 2), where all fractional dislocations are placed simultaneously on adjacent planes and are allowed to relax until they reach their equilibrium configuration; (b) all fractional dislocations corresponding to the trailing partials are pinned at the origin, while all fractional dislocations corresponding to the leading partials are allowed to relax; and (c) the placement of the leading partials occurs at different times. We find a small overlap between SFs of about $3 \mathrm{~nm}$ in (a), and a partial separation $d_{1}^{(3)}>d_{2}^{(3)} \approx d_{3}^{(3)}$. On the other hand, in case (b), the SF width is enhanced by about a factor of $2-3$, which is also in agreement with the experiment. The largest partial separation occurs in the second plane $d_{2}^{(3)}>d_{1}^{(3)}, d_{3}^{(3)}$ because it is assisted by the large elastic repulsive forces of all other leading and trailing partials on the first and third planes. Finally, in (c) we find that the average overlap of SFs increases further, consistent again with the experiment of heterogeneous nucleation, and that the largest partial separation occurs on the first plane. This is due to the fact that the leading partial on the first plane, nucleated at an earlier time, is repelled both by the leading partials on the second and third planes which are placed at subsequent times. This gives rise to a cascadelike effect where each leading partial nucleated at a later time on successive glide plane assists the widening of the SF of the previous plane, i.e., $d_{1}^{(3)}>d_{2}^{(3)}>d_{3}^{(3)}$. It is worth mentioning here that our work focuses on the widening of stacking faults in single crystals, where both leading and trailing partials are allowed to relax.
However, in contrast with our calculations, in the RAP of nanomaterials, the grain boundaries could emit the leading partial without having the trailing partial nucleated at the grain boundary. ${ }^{16}$ The pinned trailing partials are included in our calculations because the structure of the grain boundary is unknown to us. Therefore, the presence of the pinned trailing partials is assumed to compensate for the effect of grain boundaries.

In summary, we have found that the elastic interaction of partial dislocations in multiple planes leads to a widening of the SF when compared to its value for a single plane. Twin nucleation is expected to be more favorable in the case of identical partials when compared to the nonidentical case that yields zero strain. Pinning the trailing partials increases both the width of the SFs and their overlap distances on multiple planes making twinning easier compared to the bulk case.

The work at California State University, Northridge (CSUN) and UCLA were supported by NSF-NIRT under Grant No. CMS-0506841. The research at CSUN was also supported by U.S. Army under Grant W911NF-04-1-0058.

${ }^{1}$ R. Z. Valiev, I. V. Alexadrov, Y. T. Zhu, and T. C. Lowe, J. Mater. Res. 17, 5 (2002).

${ }^{2}$ X. Z. Liao, F. Zhou, E. J. Lavernia, S. G. Srinivasan, M. I. Baskes, D. W. He, and Y. T. Zhu, Appl. Phys. Lett. 83, 632 (2003).

${ }^{3}$ L. Lu, Y. Shen, X. Chen, L. Qian, and K. Lu, Science 304, 422 (2004).

${ }^{4}$ J. Schiøtz, F. D. Di Tolla, and K. W. Jacobsen, Nature (London) 391, 561 (1998).

${ }^{5}$ K. S. Kumar, S. Suresh, M. F. Chisholm, J. A. Horton, and P. Wang, Acta Mater. 51, 387 (2003).

${ }^{6}$ M. Chen, E. Ma, K. J. Hemker, H. Sheng, Y. Wang, and X. Cheng, Science 300, 1275 (2003).

${ }^{7}$ H. Van Swygenhoven, Science 296, 66 (2002).

${ }^{8}$ S. Hai and E. B. Tadmor, Acta Mater. 51, 117 (2003).

${ }^{9}$ X. L. Wu, Y. T. Zhu, and E. Ma, Appl. Phys. Lett. 88, 121905 (2006).

${ }^{10}$ V. Yamakov, D. Wolf, S. R. Phillpot, A. K. Mukherjee, and H. Gleiter, Nat. Mater. 1, 45 (2001).

${ }^{11}$ H. Van Swygenhoven, P. M. Derlet, and A. Frøseth, Nat. Mater. 3, 399 (2004).

${ }^{12}$ H. Van Swygenhoven, A. Caro, and D. Farkas, Mater. Sci. Eng., A 309, $440(2001)$.

${ }^{13}$ X. Z. Liao, F. Zhou, E. J. Lavernia, D. W. He, and Y. T. Zhu, Appl. Phys. Lett. 83, 5062 (2003).

${ }^{14}$ X. Z. Liao, Y. H. Zhao, S. G. Srinivasan, Y. T. Zhu, R. Z. Valiev, and D. V. Gunderov, Appl. Phys. Lett. 84, 592 (2004).

${ }^{15}$ S. V. Bobylev, M. Gutkin, and I. Ovid'ko, Phys. Rev. B 73, 064102 (2006).

${ }^{16}$ X. L. Wu, X. Z. Liao, S. G. Sirnivansan, F. Zhou, E. J. Lavernia, R. Z. Valiev, and Y. T. Zhu (unpublished).

${ }^{17}$ M. Shehahdeh, G. Lu, S. Banerjee, N. Kioussis, and N. Ghoniem, Philos. Mag. 87, 1513 (2007).

${ }^{18}$ G. Lu, N. Kioussis, V. Bulatov, and E. Kaxiras, Phys. Rev. B 62, 3099 (2000).

${ }^{19}$ G. Lu, V. Bulatov, and N. Kioussis, Phys. Rev. B 66, 144103 (2002).

${ }^{20}$ P. E. Blöchl, Phys. Rev. B 50, 17953 (1994).

${ }^{21}$ G. Kresse and J. Hafner, Phys. Rev. B 47, 558 (1993).

${ }^{22}$ J. P. Hirth and J. Lothe, Theory of Dislocations, 2nd ed. (Wiley, New York, 1982), p. 298.

${ }^{23}$ V. J. Wang and H. Huang, Appl. Phys. Lett. 85, 5983 (2004). 